\title{
Caracterização do Perfil Assistencial dos Pacientes Atendidos em uma Unidade de Intercorrência Oncológica
}

\author{
Caroline Nunes Pinto Fernandes, ${ }^{1}$ Maria Lia Coutinho Carvalho Ximenes, ${ }^{2}$ \\ Mirizana Alves de Almeida ${ }^{3}$
}

\begin{abstract}
RESUMO
Objetivo: Caracterizar o perfil assistencial dos pacientes atendidos em uma unidade de intercorrência oncológica. Metodologia: Pesquisa quantitativa, descritiva e documental, aprovada com o protocolo no 2.926.639. O local do estudo foi o Hospital Haroldo Juaçaba (HHJ). A população foi composta por pacientes admitidos na Unidade de intercorrência de Câncer. Foram analisados retrospectivamente os prontuários eletrônicos dos pacientes atendidos entre os meses de fevereiro e abril de 2018 na Unidade, coletando dados sociodemográficos e clínicos. Resultados: Dos cem prontuários que compuseram a amostra, identificou-se uma média de idade de 57 anos ( \pm 15 anos), sendo $68 \%$ do sexo feminino. Quanto ao estado civil, a maior parcela da amostra estudada era casada (59\%). Sobre a procedência, 65\% eram da capital e $35 \%$ do interior do Estado. Houve registro de comorbidades em $47 \%$ dos pacientes. A terapêutica mais realizada foi a quimioterapia ( $82 \%$ ). Quanto à localização primária do tumor nestes pacientes, os tumores gastrointestinais (23\%) e câncer de mama (21\%) foram os predominantes. Havia registro de complicações durante a permanência na unidade em $84 \%$ dos prontuários. Apresentando um nível de funcionalidade bem variado, $40 \%$ se mostraram independentes, $26 \%$ necessitavam de uma dependência ocasional e $34 \%$ eram totalmente dependentes. Foi identificada a solicitação em apenas $13 \%$ dos prontuários. Ocorreu, no entanto, atendimento fisioterapêutico em $55 \%$ dos pacientes da unidade. Conclusão: 0 estudo permitiu estabelecer a caracterização do perfil assistencial dos pacientes que foram atendidos na unidade de intercorrência oncológica do HHJ, oferecendo dados com potencial para contribuir com estudos futuros e prática de medidas gerenciais, tendo em vista a melhoria da qualidade e da segurança do atendimento no serviço que foi cenário do estudo.
\end{abstract}

Palavras-chave: Perfil de saúde. Neoplasias. Oncologia. Emergências.

\section{CHARACTERIZATION OF THE ASSISTANCE PROFILE OF PATIENTS \\ CARRIED OUT IN A UNIT OF ONCOLOGICAL INTERCORRENCE}

\section{ABSTRACT}

Objective: Characterize the care profile of patients treated at an oncological intercurrence unit. Methodology: Quantitative, descriptive and documentary research, approved with protocol No. 2.926.639. The study took place at Haroldo Juaçaba Hospital (HHJ). Population composed of patients admitted to the Cancer Intercurrent Unit. We retrospectively analyzed the electronic medical records of patients seen between February and April of 2018 at the Unit, collecting socio-demographic and clinical data. Results: Of the 100 charts that compose the sample, an average age of 57 years ( \pm 15 years) was identified, being $68 \%$ female. Regarding marital status, the largest portion of the sample was married (59\%). About the origin $65 \%$ were from the capital and $35 \%$ from the inland. Comorbidities were recorded in $47 \%$ of the patients. The most accomplished therapy was chemotherapy (82\%). Regarding the primary tumor location in these patients, gastrointestinal tumors $(23 \%)$ and breast cancer (21\%) were the predominant ones. There were records of complications during the stay in the unit in $84 \%$ of the medical records. With a very varied level of functionality, $40 \%$ were independent, $26 \%$ needed occasional dependence and $34 \%$ were totally dependent. The request was identified in only $13 \%$ of the medical records. However, $55 \%$ of the patients in the unit received physical therapy. Conclusion: The study made it possible to define the characterization of the care profile of patients who were treated at the oncology unit at the $\mathrm{HHJ}$, providing data with the potential to contribute to future studies and implement management measures to improve the quality and safety of care in the service which was a study scenario.

Keywords: Health profile. Neoplasms. Oncology. Emergencies.

RECEBIDO EM: 25/4/2019

MODIFICAÇÕES SOLICITADAS EM: 15/4/2020

ACEITO EM: 2/5/2020

\footnotetext{
${ }^{1}$ Autora correspondente. Instituto do Câncer do Ceará. Avenida Brasil 665, Parque das Nações, Caucaia, 61642-170, Fortaleza/CE, Brasil. http://lattes.cnpq br/8940823289968091. https://orcid.org/0000-0001-6480-3695. carolinenpinto@hotmail.com

2 Hospital Haroldo Juaçaba (HHJ). Fortaleza/CE, Brasil.

Centro Universitário Christus. Fortaleza/CE, Brasil.
} 


\section{INTRODUÇÃO}

O câncer representa um conjunto de mais de cem doenças que têm em comum o crescimento desordenado de células que invadem tecidos e órgãos. Dividindo-se rapidamente, tendem a ser muito agressivas e incontroláveis, determinando a formação de tumores malignos que podem se espalhar para outras regiões do corpo (INCA, 2018a).

No atual processo de adoecimento, o câncer ocupa uma posição de destaque na população em âmbito mundial, sendo responsável por um elevado índice de mortalidade e de internações, estando entre as quatro principais causas de morte precoce (antes dos 70 anos de idade). No Brasil, é hoje a segunda causa de morte, logo após as doenças cardiovasculares (CASTRO, 2018; INCA, 2019).

A incidência crescente de casos de neoplasia tem ocasionado uma transformação no perfil epidemiológico da população. Vários fatores explicam essa mudança no perfil do adoecimento, entre eles o aumento à exposição de fatores cancerígenos e o enveIhecimento populacional com maior expectativa de vida, além do aprimoramento das tecnologias para o diagnóstico com qualidade e melhores registros de informações. Verifica-se, também, um declínio dos tipos de câncer associados a infecções e o aumento daqueles associados à melhoria das condições socioeconômicas (BATISTA; MATTOS; SILVA, 2015; INCA, 2019).

$A$ escolha terapêutica é um determinante significativo da cura de pacientes com diversos tipos de câncer. Entre os métodos disponíveis mais realizados, destacam-se as ressecções cirúrgicas, a radioterapia, a quimioterapia e a hormonioterapia, sendo poucas as neoplasias malignas tratadas com apenas uma modalidade terapêutica. Embora essas intervenções apresentem, em muitos casos, alta eficácia na remoção e/ ou ataque a células malignas, acabam por afetar células saudáveis, desencadeando uma série de efeitos adversos que podem levar a comprometimentos agudos e/ou crônicos, afetando a qualidade de vida dos pacientes (KALIKS et al., 2017; LOPES et al., 2016).

A assistência integral pelos serviços é de suma importância para a qualidade do tratamento, posto que esses pacientes estão em risco constante de várias emergências oncológicas ameaçadoras da vida durante o tratamento. A classificação desses eventos é bem variada, podendo ser estrutural e obstrutiva (exemplo: síndrome da veia cava superior), metabólica ou hormonal (exemplo: hipercalcemia), relaciona- da ao tratamento (exemplo: síndrome de lise tumoral) ou podem ser classificados de acordo com o órgão específico atingido (KAMEO et al., 2018).

Uma parcela importante dos pacientes com câncer experimenta, pelo menos, uma emergência durante a sua doença. À medida que o tratamento avança, um maior número de pacientes pode procurar os setores de assistência à saúde, em unidades de intercorrências oncológicas, para tratar das complicações relacionadas direta ou indiretamente ao câncer e/ou por toxicidade do próprio tratamento. Muitas dessas situações de alto risco podem ser prevenidas se efetivamente diagnosticadas e prontamente reconhecidas e tratadas (BOAVENTURA; VEDOVATO; SANTOS, 2015).

No ambiente intra-hospitalar, as unidades de alta complexidade, na qual a unidade de intercorrência se enquadra, têm como finalidade essencial contribuir para a restauração da qualidade de vida dos pacientes diante das complicações apresentadas. Tradicionalmente, essas unidades consistiam de tratamento prestado essencialmente por médicos e enfermeiros, mas atualmente já existe um processo de mudança assistencial, com a inserção de uma equipe multiprofissional reestruturando os modelos de atenção à saúde (GONÇALVES et al., 2018).

A unidade de intercorrência oncológica possui uma demanda espontânea, muitas vezes maior que a prevista, decorrente de uma dinâmica intensa de atendimentos, sobrecarregando a equipe multidisciplinar desse serviço. Dessa forma, conhecer a imprevisibilidade da demanda, aliada à gravidade e à complexidade, torna esse cenário um verdadeiro desafio. Assim sendo, considerando que até este momento nenhum estudo acerca das características dos pacientes e dos cuidados prestados pela fisioterapia foi realizado na Instituição sobre esse serviço, foi delineada a presente pesquisa.

Pretende-se, com esse estudo, caracterizar o perfil assistencial dos pacientes atendidos em uma unidade de intercorrência oncológica, visando a contribuir e mostrar aos profissionais de saúde as peculiaridades dos atendimentos a essa população de pacientes, e, com isso, dar uma assistência adequada aos usuários nas diferentes situações de saúde, a partir da integração e da vinculação das diferentes áreas profissionais, pautadas na cooperação e troca de saberes.

\section{METODOLOGIA}

Esta investigação trata-se de um estudo do tipo descritivo, retrospectivo, documental e de abordagem quantitativa. Para sua execução, a pesquisa foi sub- 
metida ao Comitê de Ética e Pesquisa do Instituto do Câncer do Ceará (ICC)/Educação Continuada em Oncologia (ECO), sendo analisado e aprovado com o protocolo de $\mathrm{n}$ - 2.926.639.

O local do estudo e a coleta de dados ocorreram no Hospital Haroldo Juaçaba (HHJ)/ICC. A população foi composta por pacientes admitidos na Unidade de intercorrência de Câncer (UIC). Foram analisados retrospectivamente, durante os meses de outubro a novembro de 2018 , os prontuários eletrônicos dos pacientes atendidos entre os meses de fevereiro e abril de 2018 na Unidade. Dos prontuários que foram levantados durante esse período, foram selecionados aleatoriamente cem pacientes, perfazendo uma amostra não probabilística por conveniência.

$\mathrm{O} \mathrm{HHJ/ICC} \mathrm{é} \mathrm{uma} \mathrm{instituição} \mathrm{filantrópica} \mathrm{situada}$ no município de Fortaleza/CE. A Unidade de Intercorrência de Câncer - UIC - do HHJ oferece para pacientes do Sistema Único de Saúde (SUS) e de planos de saúde tratamento oncológico (quimioterapia, radioterapia e pós-cirúrgico) no hospital. Esse atendimento humanizado abrange procedimentos, como ajustes de medicamentos, reações adversas severas aos tratamentos e/ou aos procedimentos médicos realizados e qualquer outro tipo de alteração preocupante no quadro clínico do paciente. As indicações para admissão na unidade são: quimioterapia ou radioterapia em andamento ou terminado a menos de 30 dias; cirurgia com menos de 30 dias; sangramento ativo; trombose venosa profunda; síndrome de compressão medular e síndrome da veia cava superior.

Os dados foram coletados nos prontuários eletrônicos, avaliando o registro de informações, a fim de identificar conteúdos relevantes à pesquisa. Os dados foram coletados pelos pesquisadores por meio de uma ficha de obtenção destes, elaborada pelos mesmos, contendo questões de caracterização sociodemográfica (sexo, idade, procedência, estado civil e escolaridade) e clínica (diagnóstico, comorbidades, tratamentos realizados, tempo de permanência na unidade, dentre outros).

Após a coleta os dados foram processados pelo software Microsoft Excel 2010 e realizou-se análise por intermédio de estática descritiva e, em seguida, discutidos à luz da literatura referente ao assunto, sendo apresentados em tabelas e figuras para facilitar o entendimento e interpretação.

As informações coletadas têm a garantia do sigilo que assegura a privacidade e o anonimato dos sujeitos quanto aos dados confidenciais envolvidos na pesquisa. A pesquisa foi realizada conforme a resolução no 466/12 do Conselho Nacional de Saúde/
Ministério da Saúde, que estabelece aspectos éticos e legais da pesquisa com seres humanos. $O$ estudo respeitou os princípios fundamentais de autonomia, beneficência, não maleficência, justiça e equidade.

\section{RESULTADOS E DISCUSSÃO}

Os dados coletados possibilitaram analisar características sociodemográficas e clínicas dos atendimentos realizados aos pacientes na unidade de intercorrência oncológica do ICC.

Dos cem prontuários que compuseram a amostra, identificou-se uma média de idade de 57 anos ( \pm 15 anos), sendo $68 \%$ do sexo feminino. Os resultados encontrados nesta pesquisa divergem dos dados da estimativa de novos casos de câncer para o triênio 2020/2022, realizado pelo Instituto nacional do câncer (INCA, 2019), indicando a ocorrência de 215,86 (cem mil) em homens e 145,00 (cem mil) em mulheres, demonstrando, assim, uma perspectiva de maior incidência em homens. Na tabela 1 estão apresentadas as características sociodemográficas identificadas nos pacientes oncológicos.

Em contrapartida, cotidianamente as mulheres procuram mais os serviços de saúde, aumentando as chances de detecção precoce e tratamento acertado para as doenças. Já os homens recorrem aos serviços de saúde quando a doença está mais avançada, com seu estado de saúde bastante debilitado e, frequentemente, não têm adesão aos tratamentos propostos (GONÇALVES et al., 2018).

Verificou-se maior relato de diagnóstico com aumento da idade, corroborando os resultados de outro estudo (OLIVEIRA et al., 2015). Ressalta-se que o rápido processo de transição demográfica e suas consequências têm aumentado o peso do câncer nos dados de morbimortalidade no Brasil, uma vez que, com o envelhecimento da população brasileira, as doenças crônicas passaram a representar uma expressiva e crescente demanda aos serviços de saúde (MEDICl; BELTRÃO, 2015).

Quanto ao estado civil, a maior parcela da amostra estudada mostrou-se casada (59\%), seguida por solteiros (25\%), viúvos (9\%), divorciados $(5 \%)$ e não informado (2\%). Dado semelhante foi encontrado no estudo de Gonçalves et al. (2018). Em seu estudo, $61 \%$ dos investigados eram casados, mostrando que a família deve ser considerada aliada para a introdução das medidas de cuidado. $O$ fato de se viver com companheiro é visto como benéfico e pode influenciar no estado de saúde do portador de neoplasia, uma 
vez que a presença de um companheiro proporciona apoio social e auxilia na manutenção e continuidade do tratamento.

Sobre a procedência, $65 \%$ eram da capital e $35 \%$ do interior do Estado. Observa-se, com isso, maior incidência de câncer na região mais populosa e desenvolvida do Estado, o que pode estar associado aos hábitos de vida desta população. Este elevado número de casos de câncer pode ainda ser reflexo do maior número de diagnósticos e, consequentemente, do aumento nos registros (BOAVENTURA; VEDOVATO; SANTOS, 2015).

Em relação ao nível de escolaridade dos pacientes do estudo que informaram, $4 \%$ eram analfabetos, $32 \%$ possuíam Ensino Fundamental incompleto, 7\% com Ensino Fundamental completo, 4\% Ensino Médio incompleto e 6\% com Ensino Médio completo, demostrando que a maior parcela dos pacientes apresentou poucos anos de estudo. Segundo Bastos et al. (2018), existe uma relação entre aspectos socioeconômicos e o diagnóstico feito quando não há mais possibilidade de cura, o que pode ser resultante da dificuldade de acesso aos programas preventivos e assistenciais de saúde. A baixa escolaridade também está associada a piores padrões de cuidados à saúde.

Tabela 1 - Características sociodemográficas dos pacientes oncológicos atendidos na unidade de intercorrência oncológica

\begin{tabular}{lc}
\hline DADOS & N=100 (\%) \\
\hline $\begin{array}{l}\text { Idade (anos, média +DP) } \\
\text { Gênero }\end{array}$ & $57 \pm 15$ \\
Feminino/Masculino & $68 / 32$ \\
Estado Civil & \\
$\begin{array}{l}\text { Solteiro/Casado/Divorciado/Viúvo/Não } \\
\text { Informado }\end{array}$ & $25 / 59 / 5 / 9 / 2$ \\
$\begin{array}{l}\text { Procedência } \\
\text { Capital/Interior }\end{array}$ & $65 / 35$ \\
Escolaridade & \\
Analfabeto/EFI/EFC/EMI/EMC/Não & \\
Informado & $4 / 32 / 7 / 4 / 6 / 47$ \\
\hline
\end{tabular}

$* \mathrm{EFI}=$ Ensino Fundamental Incompleto/ EFC = Ensino Fundamental Completo $/ \mathrm{EMI}=$ Ensino Médio Incompleto/ $\mathrm{EMC}=$ Ensino Médio Completo. Fonte: As autoras.

Houve registro de comorbidades em $47 \%$ dos pacientes. As terapêuticas realizadas foram quimioterapia, radioterapia, cirurgia e hormonioterapia, representadas em $82 \%, 57 \%, 50 \%$ e $12 \%$ dos prontuários, respectivamente. Dado aproximado no estudo de Gonçalves et al. (2018), quando as terapêuticas realizadas com maior frequência nos pacientes deste estu- do foram quimioterapia (36\%) e intervenção cirúrgica (36\%). As características clínicas estão representadas na Tabela 2.

Tabela 2 - Características clínicas dos pacientes oncológicos atendidos na unidade de intercorrência

\begin{tabular}{lcc}
\hline \multicolumn{1}{c}{ DADOS } & SIM & N=100 (\%) \\
\hline \multirow{2}{*}{ Comorbidades } & 47 & NÃO \\
Quimioterapia & 82 & 53 \\
Radioterapia & 57 & 18 \\
Cirurgia & 50 & 43 \\
Hormonioterapia & 12 & 50 \\
Desfecho & ALTA & 88 \\
& 76 & ÓBITO \\
& & 24 \\
\hline
\end{tabular}

Fonte: As autoras.

As intervenções cirúrgicas, assim como a quimioterapia antineoplásica e a radioterapia, são promissoras maneiras de combater o câncer e podem ser empregadas com finalidade curativa ou paliativa. Os objetivos dos tratamentos do câncer são a cura, aumentar a sobrevida quando não há possibilidade de cura e proporcionar cuidados paliativos. À medida que a doença avança, mesmo em vigência do tratamento com intenção curativa, a abordagem paliativa deve ser ampliada visando também a cuidar dos aspectos psicológicos, sociais e espirituais (INSTITUTO ONCOGUIA, 2016; INCA, 2018b).

Acerca do desfecho, a maior frequência foi de alta para o domicílio, $76 \%$, e $24 \%$ evoluíram a óbito, informações semelhantes ao evidenciado num estudo brasileiro de Boaventura, Vedovato e Santos (2015). Esse resultado mostra que os pacientes oncológicos que procuram os serviços de intercorrência poderiam resolver demandas com menor grau de urgência em consultas de rotina, acompanhamento ambulatorial ou em serviços de atenção primária (OLIVEIRA et al., 2016).

Sobre a localização primária do tumor nestes pacientes, os tumores gastrointestinais (23\%) e câncer de mama (21\%) foram os predominantes, seguidos pelo câncer de colo do útero e os de cabeça e pescoço, como podemos observar na Tabela 3. Os dados encontrados nesta pesquisa aproximam-se dos vistos no trabalho de Boaventura, Vedovato e Santos (2015), quando os cânceres mais prevalentes foram os gastrointestinais $(27,3 \%)$, seguidos do de pulmão $(18,6 \%)$ e mama $(16,9 \%)$. 
Tabela 3 - Localização primária do tumor

\begin{tabular}{lc}
\hline Localização Primária do tumor & $\mathbf{N = 1 0 0 ~ ( \% ) ~}$ \\
\hline Gastrointestinal & 23 \\
Pulmão & 6 \\
Mama & 21 \\
Próstata & 3 \\
Cabeça e pescoço & 12 \\
Colo do útero & 16 \\
Ovário & 3 \\
Rede linfática & 4 \\
Ósseo & 3 \\
Hematológico & 3 \\
Bexiga & 2 \\
Outros* & 4 \\
\hline
\end{tabular}

* Outros: Leiomiossarcoma uterino (1), Vesícula seminal (1), Melanoma (1), Sitio primário não identificado (1).

Fonte: As autoras.

Para o Brasil, a estimativa para cada ano do triênio 2020-2022 mostra que ocorrerão 625 mil casos novos de câncer (450 mil, excluindo os casos de câncer de pele não melanoma). $O$ câncer de pele não melanoma será o mais incidente $(177 \mathrm{mil})$, seguido pelos cânceres de mama e próstata (66 mil cada), cólon e reto (41 mil), pulmão (30 mil) e estômago (21 mil) (INCA, 2019).

Havia registro de complicações durante a permanência na unidade em $84 \%$ dos prontuários, e um mesmo paciente apresentou mais de uma complicação, por isso o valor absoluto foi de 148 complicações. Verificou-se as do trato intestinal em $20,2 \%$ (30), as respiratórias em $16,2 \%$ (24), dor em $16,2 \%$ (24), alteração do estado de consciência em $12,1 \%$ (18) e as hematológicas em $8,1 \%$ (12) predominantemente, conforme demonstrado na Tabela 4.

A alta prevalência de cânceres gastrointestinais entre a população estudada pode explicar o alto índice de complicações do trato intestinal, interferindo diretamente na resposta ao tratamento. É importante identificar e corrigir os fatores relacionados ao seu desenvolvimento, os quais podem estar associados a alterações locais ou sistêmicas próprias da doença ou a efeitos colaterais de medicações (BASTOS et al., 2018).

A dor também foi uma das queixas mais relatadas entre os pacientes do estudo de Garcia et al. (2019), representando um total de $49,8 \%$. De todos os sintomas que um paciente com diagnóstico de câncer apresenta, a dor está entre os mais temidos, posto que se constitui como um fator determinante de sofrimento relacionado à doença.
A dispneia é um sintoma comum, ocorrendo em $45 \%$ a $70 \%$ dos pacientes com câncer avançado, sendo definida como uma sensação subjetiva e desconfortável de falta de ar quando a demanda de oxigênio é maior. Outra complicação pulmonar em pacientes acamados é o acúmulo de secreção pulmonar em razão da diminuição da movimentação do transporte mucociliar e enfraquecimento da tosse (ROCHA; CUNHA, 2016).

Além disso, as situações que o paciente enfrenta, como o medo da morte, separação da família, ambiente desconhecido, procedimentos invasivos e alterações funcionais, colaboram para aumentar o estado de estresse, traumas, tensão e o tempo de internação hospitalar (DIAS et al., 2017).

Tabela 4 - Complicações apresentadas durante a permanência na unidade de intercorrência

\begin{tabular}{lcc}
\hline \multicolumn{1}{c}{ Complicações } & N $\mathbf{1 4 8 *}$ & \% \\
\hline Dor & 24 & 16,21 \\
Respiratórias & 24 & 16,21 \\
Distúrbios eletrolíticos & 5 & 3,37 \\
Hematológicas & 12 & 8,10 \\
Trato Gastrointestinal & 30 & 20,27 \\
Trato Urinário & 6 & 4 \\
Estado de consciência & 18 & 12,16 \\
Sangramentos & 3 & 2 \\
Edema & 5 & 3,37 \\
Hipotermia & 3 & 2 \\
Febre & 3 & 2 \\
Desidratação & 5 & 3,37 \\
TVP & 2 & 1,35 \\
Adinamia & 3 & 2 \\
Síndrome Compressão & 2 & 1,35 \\
Medular & & \\
Lesão por Pressão & 3 & 2 \\
\hline
\end{tabular}

O mesmo prontuário pode ter apresentado mais de uma complicação. TVP: Trombose Venosa Profunda.

Fonte: As autoras.

Com relação ao tempo de permanência na unidade, obteve-se uma média de 5 dias $( \pm 4$ dias). Esse tempo prolongado pode ser justificado pela carência de unidades de atenção básica preparadas para solucionar os problemas de saúde em fase inicial. Com isso, os pacientes passam a buscar unidades especializadas em tratamento oncológico a nível terciário, que já são insuficientes ante a demanda populacional. Desta forma, as complicações vão se agravando e necessitando de maior tempo para sua recuperação. 
De acordo com Caetano e Toscano (2019), os métodos terapêuticos para o tratamento das neoplasias desencadeiam uma série de efeitos deletérios que podem levar a debilitações agudas e crônicas em razão da citotoxicidade. A sensação de fadiga é apontada como o principal efeito colateral ao tratamento em $90 \%$ dos pacientes (em quaisquer tipos de neoplasia). Esta sensação pode ser definida como uma condição de cansaço que envolve elementos comportamentais, emocionais e cognitivos, podendo comprometer o curso do tratamento.

Durante a permanência na unidade, os pacientes apresentaram-se com um nível de funcionalidade bem variado: $40 \%$ se mostraram independentes, $26 \%$ necessitavam de uma dependência ocasional e $34 \%$ eram totalmente dependentes, como observado na Figura 1. Os dados mostram que a maior parte dos pacientes necessitou de algum tipo de auxilio durante a permanência no setor. Tal dado evidencia a necessidade de uma intervenção precoce do fisioterapeuta junto a esses pacientes, objetivando a prevenção de disfunções passíveis de ocorrência durante o curso da doença e da permanência prolongada no leito (RAIMUNDO, 2015).

A permanência prolongada no leito pode desencadear a síndrome do imobilismo, que acarreta prejuízos importantes à funcionalidade do paciente. Podem ser encontradas nestes pacientes disfunções, como diminuição da amplitude de movimento e força muscular, astenia, fadiga, desenvolvimento de contraturas e deformidades ósseas e inaptidão para a realização de tarefas rotineiras (ROCHA; CUNHA, 2016).

Figura 1 - Nível de Funcionalidade

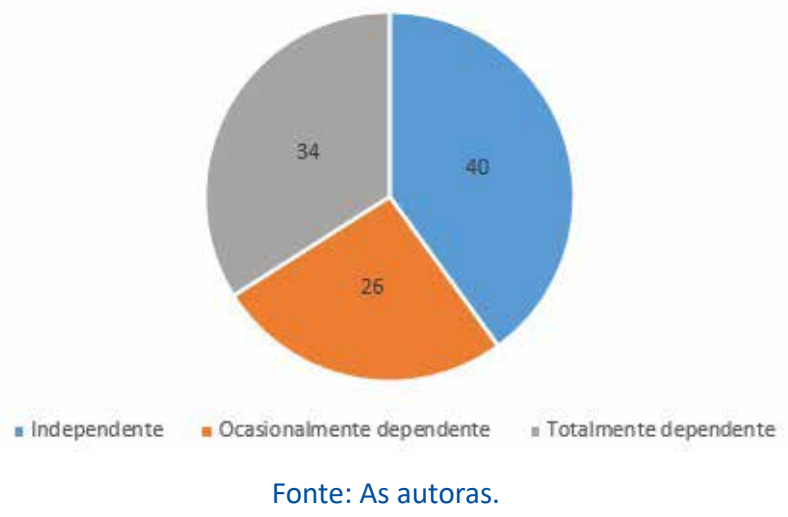

Quanto aos dados referentes à variável oxigenoterapia de apenas uma modalidade: $2 \%$ fizeram uso do cateter nasal (CN), 3\% utilizaram Máscara de Venturi (MV) e $1 \%$ a Máscara reservatório (MR). Alguns pacientes evoluíram no nível de administração dessa terapia: $3 \%$ de AA para $\mathrm{CN}, 6 \%$ de $\mathrm{AA}$ para $\mathrm{MV}, 3 \%$ de
AA para $\mathrm{CN}$ e posteriormente $\mathrm{MV}, 4 \%$ de $\mathrm{MV}$ para $\mathrm{MR}$, e $4 \%$ passaram por todos os estágios da terapia de $A A$ até MR. Apenas $1 \%$ dos pacientes conseguiu retornar da MV para AA. A maior parte dos pacientes, $73 \%$, não necessitou dessa terapia, permanecendo em ar ambiente (AA).

Segundo Frade et al. (2019), durante os dias de internação os pacientes podem apresentar um declínio funcional interferindo na sua função respiratória, podendo vir a necessitar de oxigenoterapia adicional, fator esse que pode aumentar a morbimortalidade destes indivíduos. Nesse contexto, o oxigênio constitui uma medida terapêutica em situações clínicas claras e, assim, a oxigenoterapia deve corresponder a uma prescrição adequadamente administrada.

Sobre a prescrição de fisioterapia aos pacientes atendidos na unidade durante o período do estudo, foi identificada a solicitação em apenas $13 \%$ dos prontuários. Ocorreu, no entanto, atendimento fisioterapêutico em $55 \%$ dos pacientes da unidade. 0 acompanhamento fisioterapêutico na unidade de intercorrência oncológica é realizado, em sua maioria, na presença de prescrição médica indicando o atendimento. Os fisioterapeutas, todavia, têm autonomia para que, baseados na avaliação cinético-funcional e respiratória dos demais pacientes, realizar ou não o atendimento diante das demandas apresentadas, devendo ser iniciado o mais precoce possível.

O paciente internado passa grande parte do tempo em decúbito dorsal, o que resulta em alterações na mecânica diafragmática, e, por consequência, há diminuição da ventilação. O fisioterapeuta deve estimular a adoção das posturas de sedestação e bipedestação à beira leito quando possível, além de executar e/ou orientar o paciente sobre técnicas de respiração visando a uma manutenção da capacidade vital. Além disso, os pacientes estão sujeitos aos agravos que a imobilização prolongada no leito ocasiona, tais como encurtamento muscular e rigidez articular. Desta forma, o fisioterapeuta deve realizar mobilizações e exercícios que minimizem esses efeitos adversos (FERREIRA et al., 2017).

\section{CONCLUSÃO}

O estudo permitiu estabelecer a caracterização do perfil assistencial dos pacientes que foram atendidos na unidade de intercorrência oncológica do HHJ, oferecendo dados com potencial para contribuir com estudos futuros e prática de medidas gerenciais, tendo em vista a melhoria da qualidade e segurança do atendimento no serviço que foi cenário do estudo. 
Esses resultados poderão auxiliar o planejamento dos cuidados e a atuação da equipe multidisciplinar, propiciando uma assistência mais efetiva e trazendo uma melhor qualidade e segurança no atendimento aos pacientes no hospital do estudo e de outras unidades de saúde que lidam com uma população em condição de saúde semelhante.

Percebe-se, assim, a necessidade de novas investigações científicas, considerando a carência de estudos sobre a temática apresentada, para que se possa identificar complicações mais prevalentes diante do tratamento oncológico, e, nesse contexto, elaborar estratégias de intervenção voltadas a minimizar efeitos adversos e trazendo melhores condições de saúde, além de, mais conhecimento cientifico aos profissionais que lidam diariamente com esses pacientes e auxiliar nas suas tomadas de decisão.

\section{REFERÊNCIAS}

BASTOS, B. R. et al. Perfil sociodemográfico dos pacientes em cuidados paliativos em um hospital de referência em oncologia do Estado do Pará, Brasil. Revista Pan-Amazônica de Saúde, Belém, v. 9, n. 2, p. 31-36, 2018.

BATISTA, D. R. R.; MATTOS, M.; SILVA, S. F. Convivendo com o câncer: do diagnóstico ao tratamento. Revista de Enfermagem UFSM, Rondonópolis, v. 3, n. 5, p. 499-510, set. 2015.

BoAVEnTURA, A. P.; Vedovato, C. A.; SANTOS, F. F. Perfil dos pacientes oncológicos atendidos em uma unidade de emergência. Ciencia y Enfermería, v. 21, n. 2, p. 51-62, 2015.

CAETANO, A. F. P.; TOSCANO J. J. O. Correlação entre exercício físico, qualidade de vida e níveis de fadiga nos pacientes em tratamento quimioterápico. Gepnews, Maceió, v. 2, n. 2, p. 290-298, jun. 2019.

CASTRO, A. T. A. C. T. Desmistificando as emergências oncológicas na assistência de enfermagem. Revista Eletrônica Atualiza Saúde, Salvador, v. 7, n. 7, p. 7-32, jun. 2018.

DIAS, L. B. et al. Funcionalidade e complicações em pacientes gravemente enfermos reinternados no hospital. Revista Arquivos de Ciências da Saúde, São José do Rio Preto, v. 24, n. 2, p. 60-64, jun. 2017.

FERREIRA, J. et al. Atuação do fisioterapeuta em enfermaria hospitalar no Brasil. Fisioterapia Brasil, São Luís, v. 18, n. 6, p. 788-799, 2017.

FRADE, L. et al. Oxigenoterapia na doença avançada. Medicina Interna, Lisboa, v. 26, n. 4, p. 320-325, dez. 2019.

GARCIA, J. V. M. et al. Clinical and epidemiological profile of the oncology patients in the immediate care unit. Electronic Journal Collection Health, Belém, v. 26, n. 26, jul. 2019.

GONÇALVES, M. M. et al. Perfil dos atendimentos a pacientes oncológicos em uma unidade de pronto atendimento. Revista de Enfermagem do Centro-Oeste Mineiro, 2018.
INCA. Instituto Nacional do Câncer. Estimativa 2020: incidência de câncer no Brasil. Rio de Janeiro: Instituto Nacional de Câncer José Alencar Gomes da Silva; Ministério da Saúde, 2019.

INCA. Instituto nacional do Câncer. Estimativa 2018: incidência de câncer no Brasil. Rio de Janeiro: Inca, 2018a. 128 p.

INCA. Instituto Nacional do Câncer. Tratamento do câncer. 2018b. Disponível em: https://www.inca.gov.br/tratamento/cuidados-paliativos. Acesso em: 10 mar. 2019.

INSTITUTO ONCOGUIA. Tipos de tratamento contra o câncer. 2016. Disponível em: http://www.oncoguia.org.br/conteudo/tipos-de-tratamento-contra-o-cancer/9017/1034/. Acesso em: 25 fev. 2019.

KALIKS, R. A. et al. Differences in systemic cancer treatment in Brazil. Brazilian Journal of Oncology, São Paulo, v. 44, n. 13, p. 1-12, 2017.

KAMEO, S. Y. et al. Urgências e emergências oncológica. Revista Brasileira de Cancerologia, Lagarto, v. 64, n. 4, p. 541550, 2018.

LOPES, A. B. et al. Fatores modificadores da qualidade de vida em pacientes oncológicos em tratamento quimioterápico. Revista Médica de Minas Gerais, Barbacena, v. 26, n. 3, p. 41-46, 2016.

MEDICl, A.; BELTRÃO, K. Demografia e epidemiologia do câncer no Brasil. Researchgate, maio 2015.Disponível em: https://www.researchgate.net/publication/281525086_Demografia_e_Epidemiologia_do_Cancer_no_Brasil

OLIVEIRA, M. F. et al. Estudo retrospectivo de pacientes diagnosticados com câncer de mama internados em hospital universitário. Revista Brasileira de Mastologia, Vassouras, v. 2, n. 26, p. 56-59, jan. 2016.

OLIVEIRA, M. M. et al. Estimativa de pessoas com diagnóstico de câncer no Brasil. Revista Brasileira de Epidemiologia, v. 18, n. 2, p. 146-157, dez. 2015.

RAIMUNDO, R. D. Exercícios físicos em pacientes internados em Unidade de Terapia Intensiva: novas perspectivas. $A B C S$ Health Sciences, São Paulo, v. 40, p. 2-3, 2015. Editorial.

ROCHA, L. S. M.; CUNHA, A. O papel do fisioterapeuta nos cuidados paliativos em pacientes oncológicos. JCBS, Uberaba, v. 2, n. 2, p. 78-85, 2016. 